\title{
An Undergraduate Experiment Using Cyclodextrin - Assisted Sensitive Fluorescence Detection and Quantitation of Dapsone Drug in Wastewater Samples
}

\author{
Mohammed A. Meetani, ${ }^{1, *}$, Ahmad Alhalabi ${ }^{2}$, Mohammed K. Al-tabaji ${ }^{1}$, Noor I Albadawi ${ }^{1}$, \\ Nada Elmari ${ }^{1}$, Soleiman Hisaindee ${ }^{1}$, Abdullah Al-Hemyari ${ }^{1}$, Munjed Maraqa ${ }^{2}$ \\ ${ }^{1}$ Chemistry Department, College of Science, United Arab Emirates University, P.O. Box 15551 Al-Ain, UAE \\ ${ }^{2}$ Civil and Environmental Engineering Department, United Arab Emirates University, P.O. Box 15551 Al-Ain, UAE \\ *Corresponding author: mmeetani@uaeu.ac.ae
}

Received August 10, 2019; Revised September 15, 2019; Accepted September 29, 2019

\begin{abstract}
The guest-host interaction between Dapsone drug and $\beta$-cyclodextrin ( $\beta$-CD) was investigated using fluorescence spectroscopy, ${ }^{1} \mathrm{H}-\mathrm{NMR}$, and liquid chromatography with fluorescence detection. The optimized conditions for the interaction were investigated by spectrofluorometry and were found to be at $0.2 \mathrm{mg} / \mathrm{mL}$ $(0.176 \mathrm{mM})$ of $\beta$-CD and $\mathrm{pH}$ 8.8. For these conditions, very low concentration of Dapsone drug of $2.4 \mathrm{ng} / \mathrm{mL}$ $(12.97 \mathrm{nM})$ can be detected. The standard addition method was utilized to detect Dapsone in influent and effluent wastewater samples in the sub parts per billion concentration range by HPLC-FLD using $\beta$-CD as an additive in the mobile phase.
\end{abstract}

Keywords: dapsone, $\beta$-cyclodextrin, fluorescence, guest-host interaction, wastewater

Cite This Article: Mohammed A. Meetani, Ahmad Alhalabi, Mohammed K. Al-tabaji, Noor I Albadawi, Nada Elmari, Soleiman Hisaindee, Abdullah Al-Hemyari, and Munjed Maraqa, "An Undergraduate Experiment Using Cyclodextrin - Assisted Sensitive Fluorescence Detection and Quantitation of Dapsone Drug in Wastewater Samples.” World Journal of Chemical Education, vol. 7, no. 4 (2019): 242-247. doi: 10.12691/wjce-7-4-2.

\section{Introduction}

Dapsone, also known as diaminodiphenyl sulfone (DDS), is an antibiotic drug commonly used for treatment of various skin disorders like leprosy, dermatitis and herpetiformis [1,2,3]. It has been studied extensively for pharmaceutical research such as bioavailability [4], biotransformation [5], and formulations, [6]. It has been detected in plasma, urine and saliva $[7,8]$ using different analytical methods such as liquid chromatography with, UV-Visible [8], fluorescence [9] and mass spectrometry [10], as well as electrochemical detection [8]. However, no study was found for the detection of the Dapsone drug in wastewater.

The cyclodextrins (CDs) are cyclic oligosaccharides composed of multiple subunits of glucose in an $(1,2,3,4)$ configuration. They are classified by the number of subunits ( $\alpha=6, \beta=7, \gamma=8$ ) and by the type and degree of substitution. CDs have a cavity (pore) that may accommodate small molecules as 'guests', forming inclusion complexes. The size of the pore and the environment within it can be modulated through changes to the subunits, with cavity diameters of $4.7,6.8$, and 7.5 $\AA$ for the $\alpha-, \beta-$, and $\gamma$-CD, respectively, and annular depths of 7.9-8.0 $\AA$ [11]. CDs have been used previously as a tool to enhance the fluorescence emission for a number of hydrophobic fluorophores [12,13,14]. A possible mechanism for the fluorescence enhancement is believed to be by providing favorable interactions between the fluorophore and $\mathrm{CD}$. The effect might also be derived from a reduction of the interactions between the fluorophore and water in the presence of CD [11].

CDs are used as pharmaceutical excipients, mainly as solubilizing and stabilizing agents for lipophilic substances in aqueous preparations [15,16,17]. A number of molecules are solubilized in CD solutions through formation of an inclusion complex. CDs are also known to affect the chemical stability of drug molecules. The observed effects have been extensively examined in the literature [16]. The formation of an inclusion complex usually leads to improved extraction efficiency of many chemical compounds such as antibiotics, hormones and fungicides from complex mixtures such as honey, juice or wastewater $[18,19,20]$. Wu et al. have reported the extraction and detection of fungicide, in honey and juice by solid-phase extraction using ionic-liquid-modified magnetic $\beta$-CD/attapulgite coupled with high-performance liquid chromatography (HPLC) [20]. Cui et al. have reported a new sorbent ( $\beta$-CD/ATP composite) for dispersive solid-phase extraction (d-SPE) prepared by bonding $\beta$-CD 
to modified attapulgite via silane coupling that was used to determine the concentrations of four (fluoro)quinolones (Qs) in honey samples [18]. The subsequent quantification of the Qs (ciprofloxacin, norfloxacin, ofloxacin, and gatifloxacin) was accomplished using HPLC with UV detection after employing the d-SPE procedure [18].

In this paper, the guest-host interaction between Dapsone drug and $\beta-C D$ is proposed using UV-Visible absorption, fluorescence, and NMR spectroscopy. The supramolecular complex formation effect on the fluorescence signal intensity and its influence on method sensitivity are investigated. An HPLC-FLD method that utilizes $\beta$-CD as a mobile phase modifier is developed to separate and quantitate the levels of the Dapsone drug in wastewater influent and effluent samples using the standard addition method. This method could be applied as a fourth year undergraduate experiment, offering hands-on experience in HPLC and fluorescence detection.

\section{Experimental}

Dapsone, $\alpha-, \beta$, and $\gamma$ - CDs, ethanol, acetone and chloroform (HPLC grade) were purchased from Sigma Aldrich, USA. Doubly distilled water obtained from gradient Milli-Q system (Millipore) was used to prepare stock and working solutions. Fluorescence and UV-visible absorption measurements were carried out using Agilent Cary Eclipse fluorescence spectrofluorometry (Agilent, USA) and SPCORD® 210 spectrophotometer (AnalytikJena, Germany). HPLC analysis was carried out by Agilent 1200 LC system with fluorescence detector (FLD) (Agilent, USA).

Liquid chromatographic separation was conducted on Symmetry C18 column (150 mm, $4.6 \mathrm{~mm}, 5 \mu \mathrm{m})$, (Waters, UK) at $55^{\circ} \mathrm{C}$ column temperature to achieve the chromatographic separations with isocratic elution. The injection volume was $10 \mu \mathrm{L}$. The mobile phase used was made of a (85:15) mixture of $20 \mathrm{mM}$ aqueous phosphate buffer $(\mathrm{pH}=8.8)$ and ethanol. The final concentration of $\beta$ $\mathrm{CD}$ added to the mobile phase was $5 \mathrm{mM}$. The flow rate of the mobile phase was $0.3 \mathrm{ml} / \mathrm{min}$. The fluorescence detection wavelengths were set at $\lambda$ ex $=292 \mathrm{~nm}$ and $\lambda \mathrm{em}=428 \mathrm{~nm}$.

a stock solution of $1 \mathrm{mM}$ Dapsone was prepared in deionized water and it was kept in a refrigerated dark vial. The experimental samples (working) solutions were prepared fresh daily from the stock solution. A stock solution of $\beta$-CD was prepared in deionized water and it was kept at room temperature.

UV-visible spectroscopy measurements were conducted to determine the best excitation wavelength of Dapsone for fluorescence measurements.

For the fluorescence measurements, the concentration of CD was fixed at $1.0 \mathrm{mg} / 5 \mathrm{~mL}(1.76 \mathrm{mM})$, and Dapsone concentration was varied. The $\mathrm{pH}$ was varied by adding aliquot amounts of $\mathrm{HCl}$ and $\mathrm{KOH}$ solutions and then measured using a WTW 330i pH meter with SenTix Mic glass electrode in order to reach the best detection conditions.

Analysis of Dapsone in wastewater samples, obtained from Al-Saad wastewater treatment facility, Al-Ain city, UAE, was performed after the sample was concentrated 1000 times using solid phase extraction (SPE), with SPE-DEX 4790 automated extraction system (Horizon
Technology, Salem, USA). SPE was performed using Atlantic ${ }^{\circledR}$ HLB-M disks containing N-vinylpyrrolidone and divinylbenzene sorbent (Horizon Technology, Salem, USA). Each extracted sample (20 mL) was then evaporated to dryness under the flow of nitrogen gas. The sample extract was reconstituted in (85:15) (water: ethanol) solution and divided into 5 portions in separate 5 vials, $0.2 \mathrm{~mL}$ each. A standard solution of Dapsone, $100 \mathrm{ppb}$, was prepared and spiked into these five vials with different volumes $(0.0,0.1,0.2,0.3$, and $0.4 \mathrm{~mL})$. The total volume in each vial was completed to $1 \mathrm{~mL}$ with water ethanol solution. Samples were then analyzed on the HPLC-FLD instrument.

Proton NMR measurements were carried out using Varian, $400 \mathrm{MHz}$ instrument. NMR spectra were collected for the $\beta$-CD and Dapsone separately in $\mathrm{D}_{2} \mathrm{O}$ solvent, then as a mixture of $1.54 \mathrm{mM}$ Dapsone and $3.23 \mathrm{mM} \beta-\mathrm{CD}$ in $\mathrm{D}_{2} \mathrm{O}$.

\section{Results and Discussion}

Dapsone shows two absorbance peaks at $255 \mathrm{~nm}$ and $292 \mathrm{~nm}$. Comparison of the UV-Visible spectra for the drug alone and the drug \& $\beta$-CD shows no change in absorbance intensity of Dapsone, see Figure 1. However, it was noticed that there was a red shift in the absorption maximum and a formation of an isobestic point upon the addition of the $\beta$-CD to the drug solution indicating an interaction between Dapsone drug and $\beta$-CD.

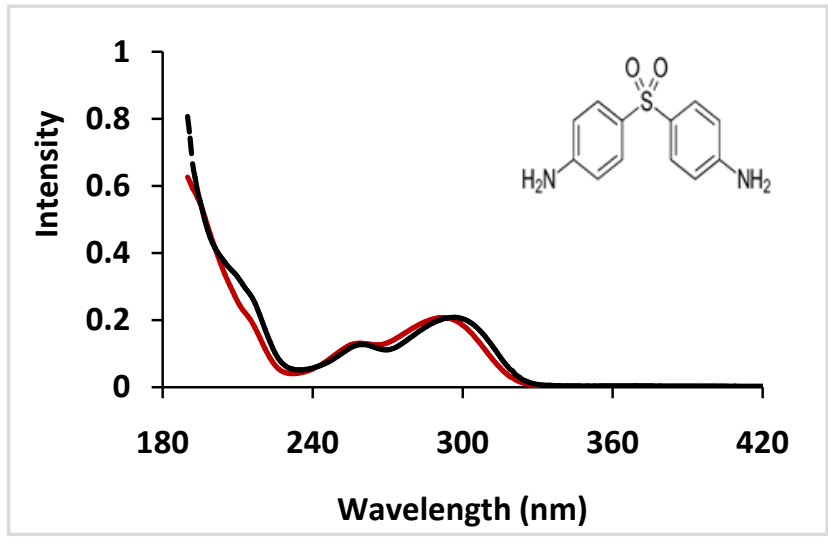

Figure 1. UV-Vis spectra of Dapsone in aqueous solution without adding $\beta$-CD (solid red line) and after adding $\beta$-CD (dotted black line). Dapsone structure is shown at the figure top

Fluorescence spectrum of Dapsone shows a low intensity peak at $460 \mathrm{~nm}$ when it is excited at $292 \mathrm{~nm}$. However, when $\beta$-CD is added to the Dapsone solution, the fluorescence of Dapsone is enhanced and the intensity is increased. Figure 2a shows the fluorescence of Dapsone without $\beta$-CD. As the concentration of the Dapsone decreased from $2 \times 10^{-5} \mathrm{M}$ to $2.04 \times 10^{-6} \mathrm{M}$ the fluorescence intensity decreased dramatically. However, when CD was added, fluorescent signal increased (see Figure 2b). It is believed that Dapsone molecules are encapsulated inside the $\beta$-CD cavity due to hydrophobic - hydrophobic interaction between the phenyl groups of Dapsone and the $\beta$-CD internal cavity. Upon guest-host complexation the Dapsone will change its surrounding environment from polar to nonpolar and as a result its fluorescence intensity gets enhanced. 

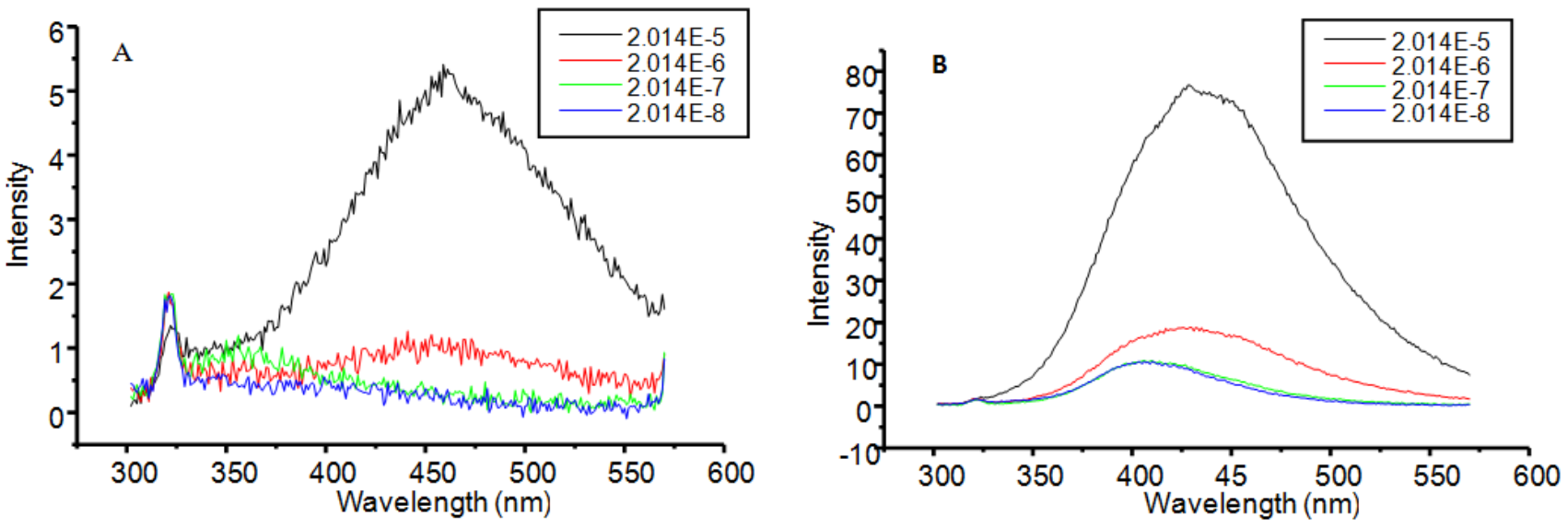

Figure 2. Fluorescence spectra of Dapsone at different concentrations (2.014 x $\left.10^{-5}-2.014 \times 10^{-8} \mathrm{M}\right)$ (A) without and (B) with $\beta$-CD

\subsection{Effect of $\mathbf{p H}$}

The $\mathrm{pH}$ of Dapsone solution was changed to acidic, basic and neutral, then the absorbance and fluorescence measurements were collected under these different conditions. Figure 3a shows the absorbance spectra for Dapsone without $\beta$-CD at different $\mathrm{pH}$ values. There was an increase in the intensity of the absorbance peak of Dapsone when its solution is made basic. Under this condition the Dapsone molecule is mostly neutral, with the two amine groups without a charge. Moreover, this condition will also enhance the $n \rightarrow \pi^{*}$ transition relative to the absorbance peak at $292 \mathrm{~nm}$ which is due to $\pi \rightarrow \pi^{*}$. An explanation for this observation is that the lone pair of the amine groups will not be available for the $n \rightarrow \pi *$ transitions in acidic conditions since they will be used to bind the $\mathrm{H}^{+}$ion available in the solution [21]. On the other hand, when the absorbance measurement was collected for Dapsone in acidic solution, the absorbance at $292 \mathrm{~nm}$ was more than that at $255 \mathrm{~nm}$. The former absorbance peak at $292 \mathrm{~nm}$ was not affected by the excess protons in the solution therefore its intensity did not change in comparison with the peak at $255 \mathrm{~nm}$ which decreased markedly. Figure $3 \mathrm{~b}$ shows the absorbance spectra of Dapsone $+\beta$-CD in three solutions of different $\mathrm{pH}$ values. $\beta$-CD did not show any absorption, while the Dapsone absorbance was almost the same under the three different conditions.

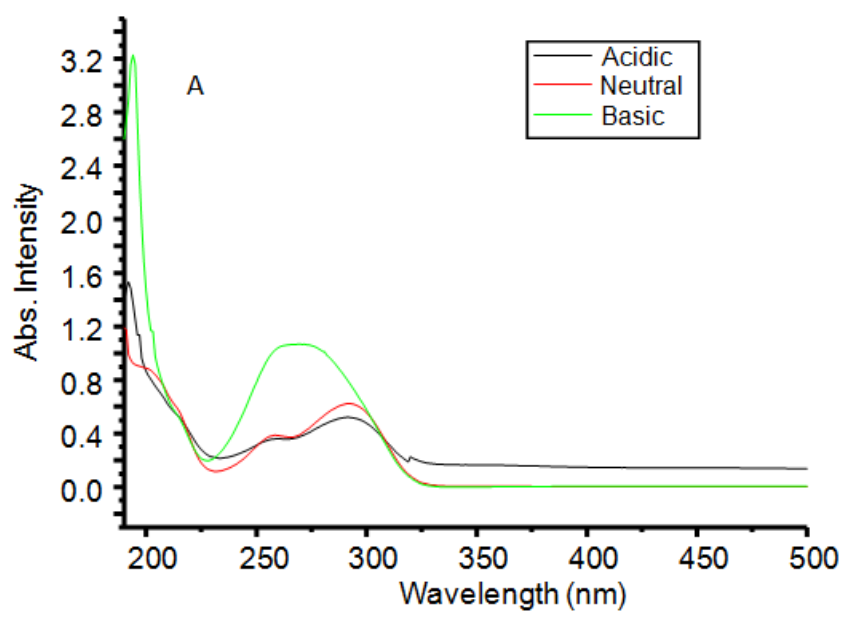

Figure 4 shows fluorescence spectra of Dapsone at low concentration at different $\mathrm{pH}$ values. Almost no effect was observed for the change in $\mathrm{pH}$ on the fluorescence intensities of the drug. However, when $\beta$-CD was added, there was a substantial increase of the fluorescence intensity of Dapsone, an increase by almost 10 times (Figure 5). This observation could be explained by the fact that $\beta$-CD will provide a non-polar microenvironment for the non-polar Dapsone drug through inclusion inside the cavity of the host $\beta$-CD which will also limit the movement of the drug molecule and add more rigidity to Dapsone drug.

The fluorescence spectra of the same concentration of Dapsone drug in the presence of $\beta-C D$ in acidic, neutral and basic solutions are shown in Figure 5, in which the fluorescence intensity of Dapsone in the basic solution gave the highest intensity while that in the acidic solution was the lowest. This could be due to the protonation of the amine groups when the solution is made acidic and consequently lower the possibility of encapsulation of the Dapsone inside the cavity of the $\beta$-CD since the drug will prefer the aqueous solution whenever it is in the ionized state and will prefer encapsulation whenever it is in the neutral state. Nonetheless, the fluorescence intensity of Dapsone under acidic conditions with $\beta$-CD is still higher than the one without adding $\beta-C D$, indicating that there is still a guest-host inclusion happening but to a lower extent than that under basic conditions.

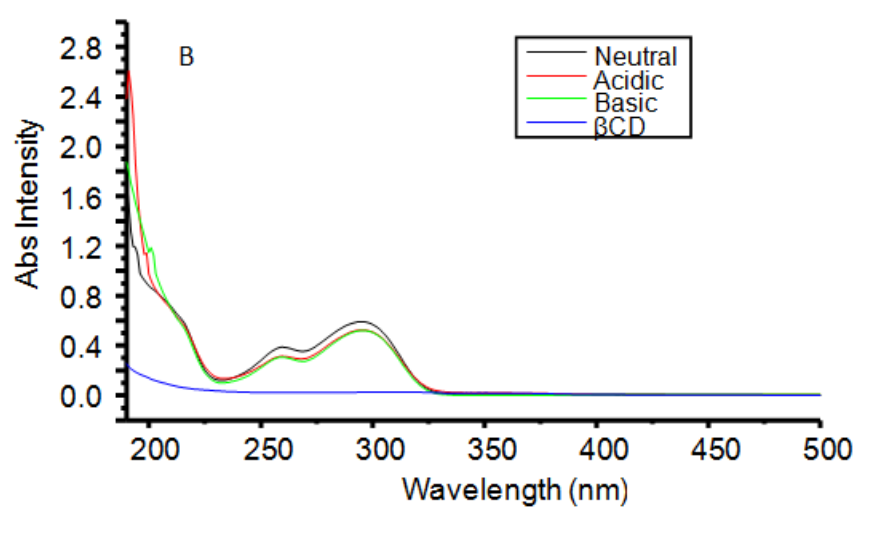

Figure 3. (a) Absorbance spectra for Dapsone without $\beta-\mathrm{CD}$ at different $\mathrm{pH}$ values. (b) Absorbance spectra for Dapsone with $\beta$-CD at different $\mathrm{pH}$ values 


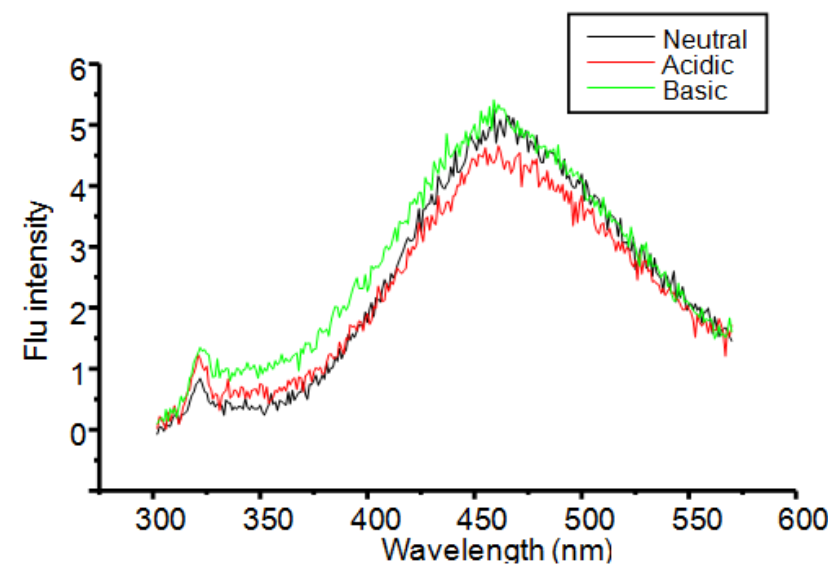

Figure 4. Fluorescence spectra of Dapsone drug in acidic, neutral and basic solutions ([Dapsone] $=2.0 \times 10^{-6} \mathrm{M}$ )

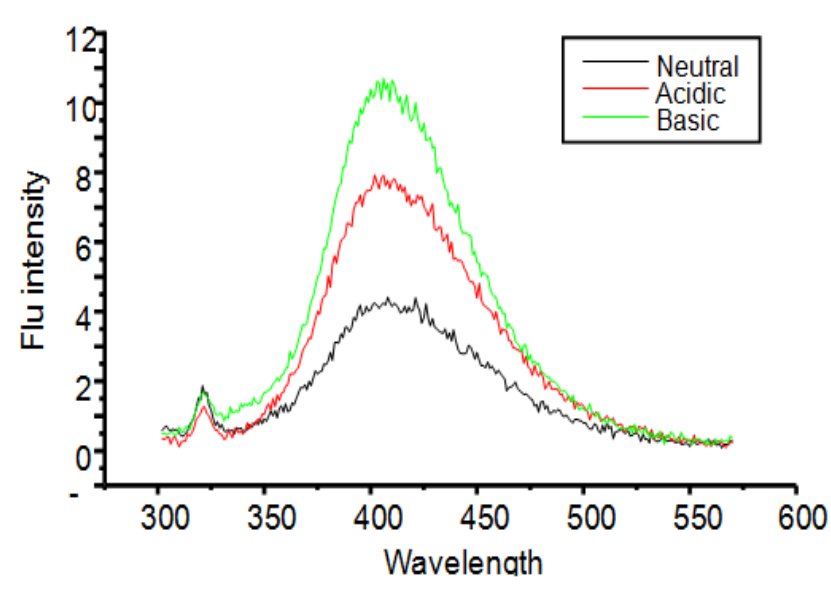

Figure 5. Fluorescence spectra of Dapsone and $\beta-C D$ in acidic, neutral, and basic solutions $\left([\right.$ Dapsone $]=2.0 \times 10^{-7} \mathrm{M}$. $\left.[\beta-\mathrm{CD}]=0.176 \mathrm{mM}\right)$
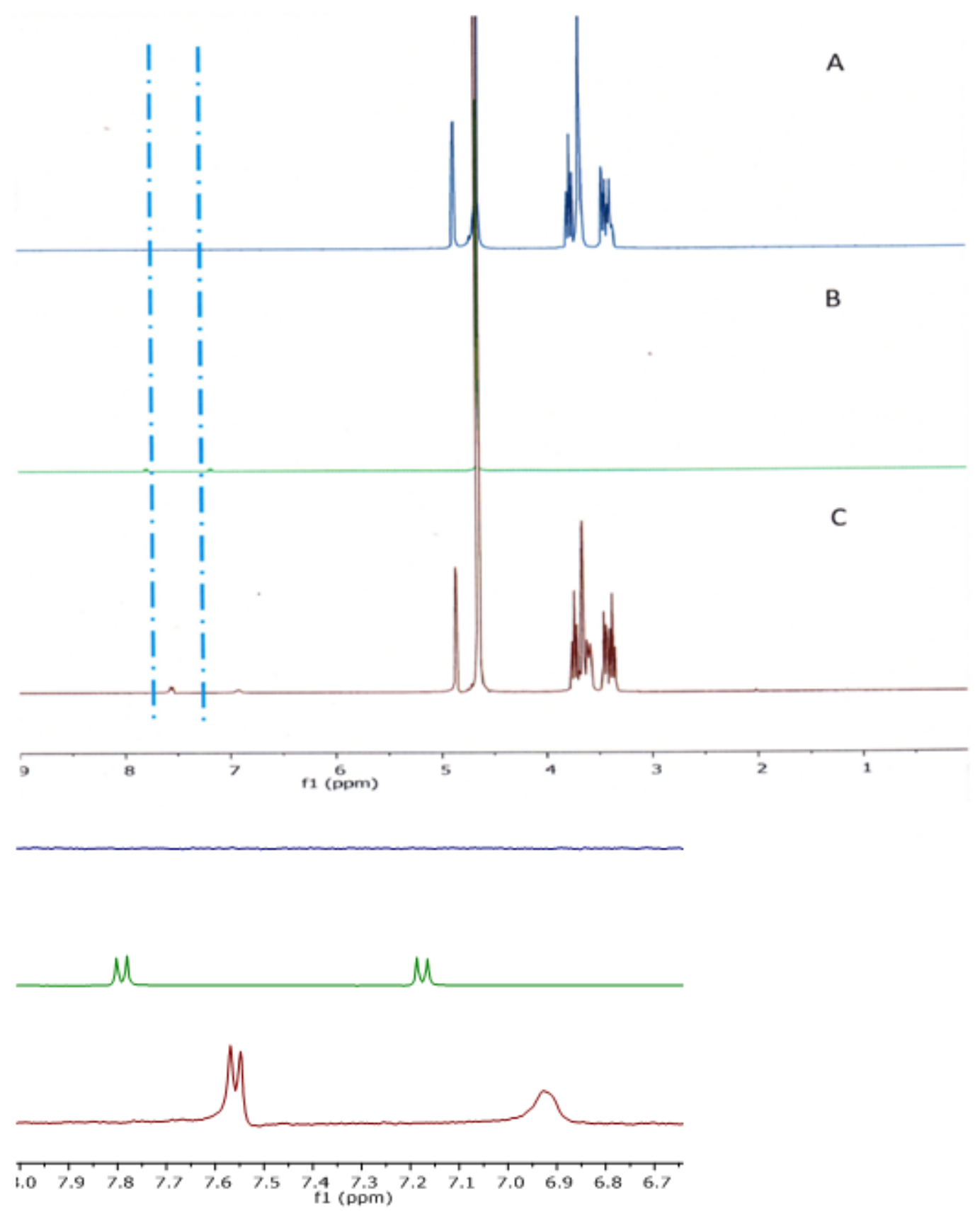

Figure 6. ${ }^{1} \mathrm{H}-\mathrm{NMR}$ spectra for (A) $2.93 \mathrm{mM} \beta$-CD, (B) $1.54 \mathrm{mM}$ Dapsone and (C) $1.54 \mathrm{mM}$ Dapsone- and $3.23 \mathrm{mM} \beta-\mathrm{CD}$ mixture (top figure). $\mathrm{D}_{2} \mathrm{O}$ was used as solvent in all the experiments. A close up to the NMR signal (6.6-8.0 ppm) is shown in the bottom figure 


\subsection{Dapsone - $\beta$-CD Interaction}

The interaction of Dapsone with $\beta-C D$ was investigated using ${ }^{1} \mathrm{H}-\mathrm{NMR}$ spectroscopy. Figure 6 shows the NMR spectra for the $\beta-C D$, Dapsone and Dapsone- $\beta-C D$ mixture. It was noticed that there is a shift of the phenyl group protons to upfield as a result of encapsulation of the Dapsone inside the $\beta$-CD cavity.

The binding constant $(\mathrm{k})$ of the supramolecular complex of Dapsone and $\beta$-CD was calculated [22] from the absorption measurements of the complex formed during the titration of Dapsone with $\beta-C D$, Figure 7 . The increase of the concentration of $\beta-C D$ enhanced the relative absorbance of the Dapsone until it reaches saturation. The binding constant between Dapsone and $\beta-C D$ was found to be 24238 , which indicates a very good interaction. The supramolecular complex of $\beta$-CD-Dapsone is formed through 1:1 stoichiometry [14].

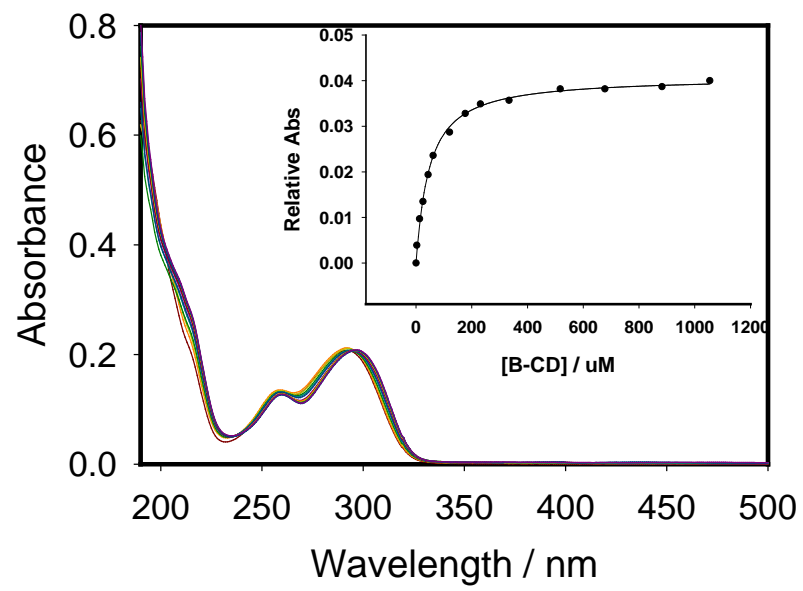

Figure 7. Absorption spectra between Dapsone and $\beta$-CD in a basic medium ( $\mathrm{pH} 10)$. Data fit for binding constant calculation is shown in the inset $\left(\mathrm{k}=24238.8204, \mathrm{~S}=4630.4750, \mathrm{R}^{2}=0.9964\right)$

\subsection{Application}

As an application for the Dapsone - $\beta$-CD interaction, an HPLC method was developed to detect Dapsone in wastewater. Initially external calibration curves for Dapsone were constructed with and without adding the $\beta-C D$ as modifier to the mobile phase. It was noted that the slope of the calibration curve has been improved significantly, - an increase of one order of magnitude was observed, when $\beta$-CD was added to the mobile phase, see Figure 8.

Since wastewater is considered a complex mixture, the possibility of detecting Dapsone without interferences is low. Therefore, a standard addition calibration curve was investigated and utilized to estimate the levels of Dapsone drug in wastewater influent and effluent samples collected from Al Saad Wastewater Treatment Plant in Al Ain, UAE. One liter each of influent and effluent wastewater samples was extracted and concentrated separately and collected in $1 \mathrm{~mL}$ vial which was dried until dryness. The sample was reconstituted in (85:15) (water: ethanol) solution and divided into 5 portions in separate 5 vials. A standard solution of Dapsone, with a concentration of 100 $\mathrm{ppb}$, was prepared and spiked into these five vials with different volumes $(0.0,0.1,0.2,0.3$, and $0.4 \mathrm{~mL})$. The total volume in each vial was topped to $1 \mathrm{~mL}$ with water ethanol solution. Samples were then analyzed on HPLCFLD. Figure 9 shows the standard addition calibration curve for the influent and effluent samples. Three replicates were measured for each wastewater sample.
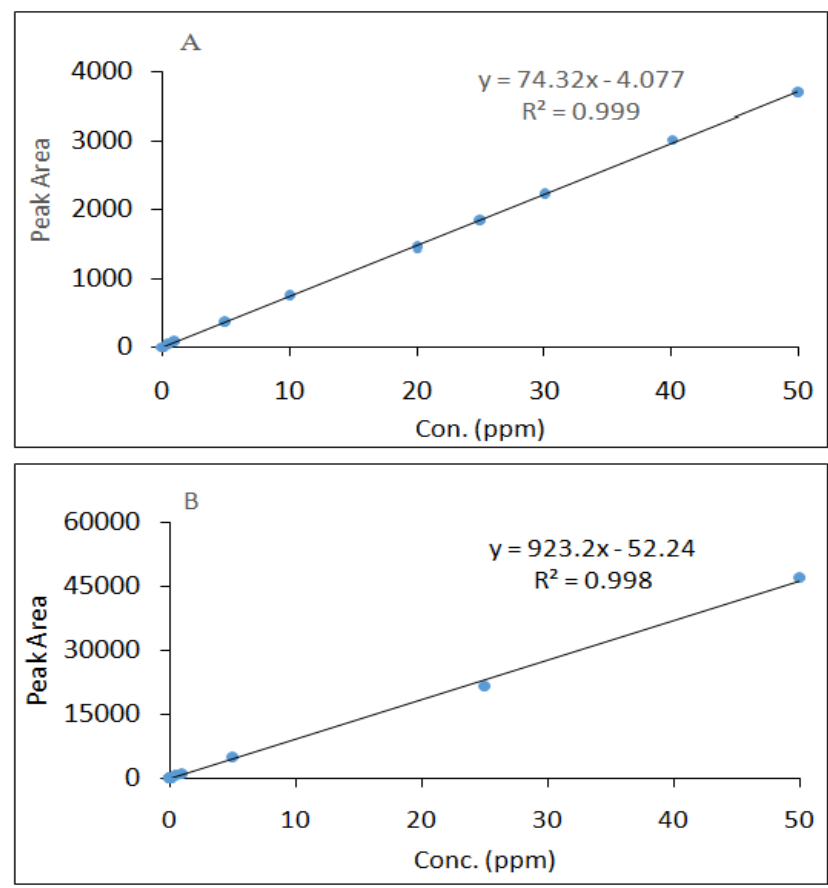

Figure 8. Calibration curve for Dapsone analyzed on HPLC-FLD (a) without $\beta-C D$, (b) after adding $\beta$-CD to the mobile phase
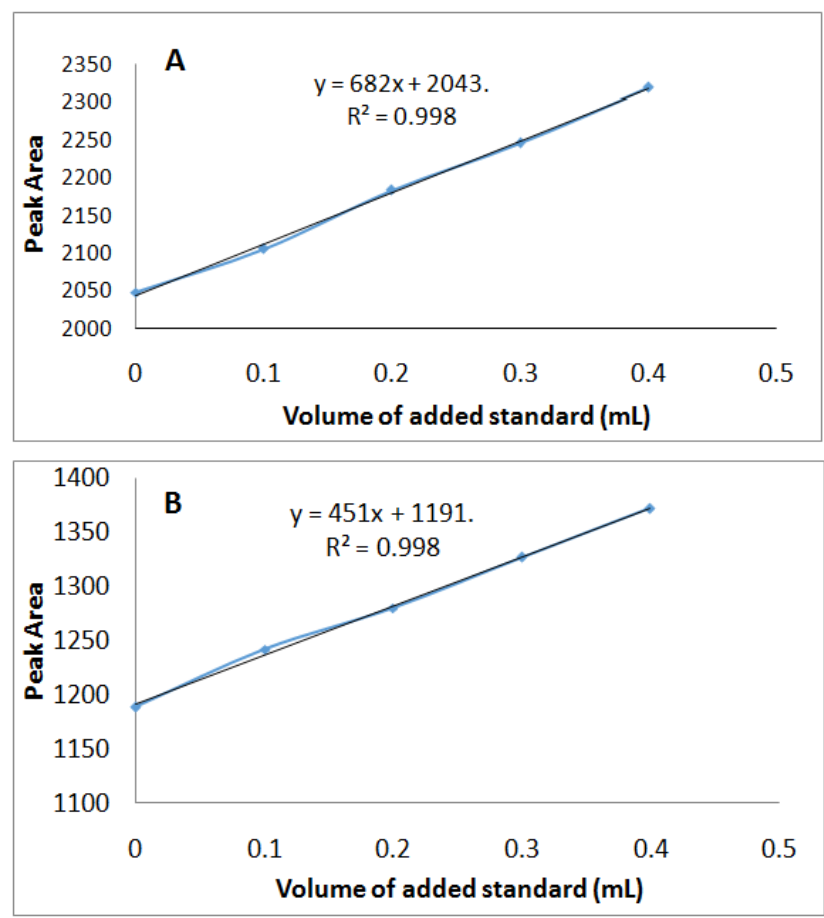

Figure 9. Standard addition calibration curve for Dapsone analyzed on HPLC-FLD (a) without $\beta$-CD, (b) after adding $\beta$-CD to the mobile phase. The unknown volume was $0.2 \mathrm{~mL}$ and the concentration of spiked Dapsone standard was $100 \mathrm{ppb}$ and its volume was $0.1 \mathrm{~mL}$

The average concentration for the Dapsone drug in the extracted influent wastewater was $1.65 \pm 0.19 \mathrm{ppb}$ and the extracted effluent wastewater contained $1.3 \pm 0.07 \mathrm{ppb}$ Dapsone. Therefore, the actual concentration of Dapsone 
in the influent wastewater is $1.65 \times 10^{-3} \mathrm{ppb}$ and in the effluent is $1.3 \times 10^{-3} \mathrm{ppb}$ since samples were concentrated from 1.0 L.

\section{Conclusion}

In this paper, we demonstrated the effect of the microenvironment on the fluorescence behavior of Dapsone drug through a guest -host interaction. Solid phase extraction was used for extraction and concentration of the wastewater samples prior to HPLC-FLD analysis. We utilized the signal enhancement of Dapsone in the presence of $\beta$-CD to develop a sensitive method of detection of the drug in complex matrix of wastewater.

\section{Acknowledgements}

This project was funded by the Research Office of the United Arab Emirates University [Fund \# 31S281].

\section{References}

[1] Zhu YI, Stiller MJ. Dapsone and sulfones in dermatology: overview and update. Journal of the American Academy of Dermatology, 45(3),420-434, 2001.

[2] Wozel G, Blasum C. Dapsone in dermatology and beyond. Archives of dermatological research,306(2), 103-124, 2014.

[3] Zampeli E, Moutsopoulos HM. Dapsone: an old drug effective for subacute cutaneous lupus erythematosus.Rheumatology (Oxford) 58(5), 920-921, May 2019.

[4] do Amaral LH, do Carmo FA, Amaro MI, de Sousa VP, da Silva L, de Almeida GS, et al. Development and Characterization of Dapsone Cocrystal Prepared by Scalable Production Methods. AAPS PharmSciTech. , 19(6), 2687-2699, 2018.

[5] van de Steeg E, Schuren FHJ, Obach RS, van Woudenbergh C, Walker GS, Heerikhuisen M, et al. An Ex Vivo Fermentation Screening Platform to Study Drug Metabolism by Human Gut Microbiota. Drug Metabolism and Disposition, 46(11), 1596-1607, Nov, 2018;

[6] Braun DE, Griesser UJ. Supramolecular Organization of Nonstoichiometric Drug Hydrates: Dapsone. Frontiers in Chemistry, 6, 31, Feb 2018.

[7] Essousi H, Barhoumi H. Electroanalytical application of molecular imprinted polyaniline matrix for dapsone determination in real pharmaceutical samples. Journal of Electroanalytical Chemistry, 818. 131-139, 2018.

[8] Moncrieff J. Determination of dapsone in serum and saliva using reversed-phase high-performance liquid chromatography with ultraviolet or electrochemical detection. Journal of
Chromatography B: Biomedical Sciences and Applications, 654(1), 103-110, 1994.

[9] Farthing CA, Farthing DE, Koka S, Larus T, Fakhry I, Xi L, et al. A simple and sensitive HPLC fluorescence method for determination of tadalafil in mouse plasma. Journal of chromatography $B$, Analytical technologies in the biomedical and life sciences, 878(28), 2891-2895, 2010.

[10] Rocha DG, Santos FA, Gomes AA, Faria AF. Validation of a LCMS/MS Multiresidue Methodology Based on a QuEChERS Approach for the Determination of Fluoroquinolones, Sulfonamides and Trimethoprim in Poultry and Porcine Kidney According to the Normative Instruction 24/2009-MAPA \%J Journal of the Brazilian Chemical Society,28, 76-86, 2017.

[11] Meyer AG, Easton CJ, Lincoln SF, Simpson GW. $\beta$-Cyclodextrin as a Scaffold for Supramolecular Chemistry, To Reverse the Regioselectivity of Nitrile Oxide Cycloadditions. The Journal of Organic Chemistry, 63(24), 9069-9075, 1998.

[12] Dong N, Cheng L-n, Wang X-l, Li Q, Dai C-y, Tao Z. Significant fluorescence enhancement by supramolecular complex formation between berberine chloride and cucurbit(n\&\#xa0;=\&\#xa0;7)uril and its analytical application. Talanta, 84(3), 684-689, 2011.

[13] Baglole KN, Boland PG, Wagner BD. Fluorescence enhancement of curcumin upon inclusion into parent and modified cyclodextrins Journal of Photochemistry and Photobiology A: Chemistry, 173(3), 230-237, 2005.

[14] Ma L, Tang B, Chu C. Spectrofluorimetric study of the $\beta$-cyclodextrin-dapsone-linear alcohol supramolecular system and determination of dapsone. Analytica Chimica Acta, 469(2), 273-283, 2002.

[15] Skiba M, Wouessidjewe D, Puisieux F, Duchêne D, Gulik A. Characterization of amphiphilic $\beta$-cyclodextrin nanospheres. International Journal of Pharmaceutics, 142(1), 121-124, 1996.

[16] Loftsson T, Brewster ME. Pharmaceutical applications of cyclodextrins. 1. Drug solubilization and stabilization. Journal of Pharmaceutical Science. , 85(10), 1017-1025, 1996.

[17] Stella VJ, Rajewski RA. Cyclodextrins: their future in drug formulation and delivery. Pharmacuetical Research, 14(5), 556-567, 1997.

[18] Cui X, Zhang P, Yang X, Yang M, Zhou W, Zhang S, et al. beta$\mathrm{CD} / \mathrm{ATP}$ composite materials for use in dispersive solid-phase extraction to measure (fluoro)quinolone antibiotics in honey samples. Analytica Chimica Acta 878, 131-139, 2015.

[19] Kopperi M, Riekkola ML. Non-targeted evaluation of selectivity of water-compatible class selective adsorbents for the analysis of steroids in wastewater. Analytica Chimica Acta. 920, 47-53, 2016.

[20] Wu X, Yang M, Zeng H, Xi X, Zhang S, Lu R, et al. Effervescence-assisted dispersive solid-phase extraction using ionic-liquid-modified magnetic beta-cyclodextrin/attapulgite coupled with high-performance liquid chromatography for fungicide detection in honey and juice. Journal of seperation Science, 39(22), 4422-4428, 2016.

[21] Kongsted J, Osted A, Mikkelsen KV, Astrand PO, Christiansen O. Solvent effects on the $n-->$ pi* $^{*}$ electronic transition in formaldehyde: a combined coupled cluster/molecular dynamics study. Journal of Chemical Physics. 121(17), 8435-8345, 2004.

[22] Thordarson P. Determining association constants from titration experiments in supramolecular chemistry. Chemical Society reviews. 40(3):1, 305-233. 2011

C The Author(s) 2019. This article is an open access article distributed under the terms and conditions of the Creative Commons Attribution (CC BY) license (http://creativecommons.org/licenses/by/4.0/). 